\title{
A METRIC SCRUTINY OF THE ORBITAL MARGINS
}

\author{
Muthusamy AkshayaNarayanan ${ }^{1}$, HannahSugirthabai RajilaRajendran *2, Gnanasundaram \\ Vaithianathan ${ }^{3}$, Govindaraju Soundararajan ${ }^{4}$, Sundaramurthi Indumathi ${ }^{5}$. \\ ${ }^{1}$ III BDS, Chettinad Dental college \& Research Institute, IT highway, Kelambakkam, Kanchipuram dist. Tamil Nadu, \\ India. \\ ${ }^{* 2}$ Professor, Department of Anatomy, Chettinad Hospital \& Research Institute, IT highway, Kelambakkam, Kanchipuram \\ dist. Tamil Nadu, India. \\ ${ }^{3}$ Assistant Professor, Department of Anatomy, Chettinad Hospital \& Research Institute, IT highway, Kelambakkam, \\ Kanchipuram dist. Tamil Nadu, India. \\ ${ }^{4}$ Professor Emeritus, Chettinad Academy of Research \& Institute, Kelambakkam, Kanchipuram dist. Tamil Nadu, \\ India. \\ ${ }^{5}$ Head \& Professor, Department of Anatomy, Chettinad Hospital \& Research Institute, IT highway, Kelambakkam, \\ Kanchipuram dist. Tamil Nadu, India.
}

\section{ABSTRACT}

Background: The anatomical boundaries of the orbit are defined and the contribution of various bones in making up the orbital margins is determined .Associated with this, orbital dimensions like length, breadth and the orbital index are determined along with the associated dimorphic and bilateral variations. The findings of these territorial specimens are analyzed and compared with similar findings from other vernacular territories. The bilateral craniofacial structures are studied and they are dimensionally classified and characterized. Comprehensive documentation thus promulgated functions to prove vital in the conquest of dexterity in the field of surgery and forensic anthropometry alike. The evaluated parameters also serve as an empirical guide in discerning tenuous sites that are susceptible to impaction and in defining the safe limits of orbital exploration Results: The study has returned an average value of 81.47 as the orbital index of the scrutinized dry skull specimens thus placing them under the category of microseme. The average values of orbital length and breadth were found to be $31.26 \mathrm{~mm}$ and $38.37 \mathrm{~mm}$ respectively. The superior margin formed exclusively by the frontal bone measured $32.7 \mathrm{~mm}$ on average with the supraorbital notch/foramen being displaced $5.92 \mathrm{~mm}$ from the medial limit of the superior margin. For $25.88 \mathrm{~mm}$, which was the average length of medial margin, maxilla stood the dominant contributor $(13.81 \mathrm{~mm})$ with the frontal bone chipping in to contribute to the rest. The inferior margin paints a contrary picture of maxilla being the secondary contributor $(16.13 \mathrm{~mm})$ whilst here it is the zygoma which makes a greater contribution $(17.29 \mathrm{~mm})$.

Conclusion: This study hereby establishes Microseme to be the predominant orbital index of the scrutinized skull specimens in addition to quantifying the contributions of various bones towards making up the margins. Comparative analysis with confluent such studies from other provinces of the Indian subcontinent has confirmed the latitude variation associated with orbital index. The results thus established will be unerringly essential in the field of surgery and forensic anthropometry.

KEY WORDS: Frontal bone, zygomatic bone, maxilla, supraorbital notch/foramen, orbital index, Microseme, Mesoseme, Megaseme.

Address for Correspondence: Dr. HannahSugirthabai RajilaRajendran, Professor, Department of Anatomy, Chettinad Hospital \& Research Institute, IT highway, Kelambakkam, Kanchipuram dist. Tamil Nadu, India. Pincode -603103, Phone number - +919710403803 E-Mail: ashrajsanada@gmail.com

Access this Article online

Quick Response code

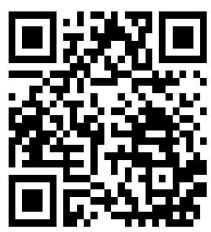

DOI: $10.16965 /$ ijar.2018.108
Journal Information

International Journal of Anatomy and Research

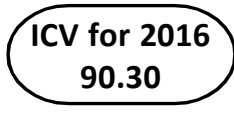

ISSN (E) 2321-4287 | ISSN (P) 2321-8967

https://www.ijmhr.org/ijar.htm

DOI-Prefix: https://dx.doi.org/10.16965/ijar

Article Information

Received: 04 Jan 2018

Peer Review: 04 Jan 2018

Revised: 19 Feb 2018
Accepted: 07 Mar 2018

Published (O): 05 Apr 2018

Published (P): 05 Apr 2018 


\section{INTRODUCTION}

The orbits are bilaterally placed pyramidal bony cavities which house contents of cardinal sapience like the eyeball, associated nerves and vessels and the extraocular muscles with the connective tissue system and fat. This anatomical space with its capacity approximated at 30cc, is bounded superiorly by the anterior cranial fossa, medially by the nasal cavity and ethmoidal air sinuses, inferiorly by the maxillary sinus and laterally by the middle cranial fossa and the temporal fossa.

The orbital cavities of dry skull specimens displaying provincial nativity were analyzed with regards to orbital length, breadth and the metric contribution of various bones towards making up the orbital margins; the orbital index was computed from the formerly mentioned values while the bilateral and dimorphic variations were inferred as well. A deep-seated comprehension of orbital anatomy is an empirical guide for surgical proficiency pertaining to orbital rim osteotomy, orbital enucleation and decompression, maxillofacial reconstruction surgeries, administration of supraorbital nerve block and preoperative planning for postoperative management. The sapience of the bony orbit in the fields of anthropometry and forensic science is inarguably profound. The characterization of regional morphometry is also of immense values to commercial manufacturers of glasses as it per se aids them in pigeonholing the gross dimensions of rims suited for a section of population.

One of the criteria for classifying the orbits is orbital index. It is defined as:

Orbital index: [orbital length/orbital breadth] *100

Based on the obtained values, the studied skulls are henceforth grouped as follows [1]:

Microseme: The orbital index is less than or equal to 83 and is commonly seen in black races where the orbit is rectangular.

Mesoseme: The orbital index is between 89 and 83 and is typical of white races like the English and European.

Megaseme: The orbital index is 89 or over and is typically observed in yellow races except the
Eskimos.

In addition to the above mentioned metric measurements, the anatomical makeup of the orbital rims is perused profoundly. Located bilaterally, each of the orbital rims serves as a base to the pyramidal orbital cavity-superior, medial, inferior and lateral margins encompassing its confines. While the bones contributing to its formation are ubiquitously homogenous, the individual contribution of each boned varies and this difference is computed. The bones making up the margins are elucidated below:

Superior margin- Frontal bone

Medial margin-Frontal bone and maxilla

Inferior margin-Maxilla and zygomatic bone

Lateral margin- Zygomatic bone and frontal bone.

\section{MATERIALS AND METHODS}

Seventy five skulls are taken for study and judged under the following parameters: Orbital length: maximum vertical distance between the upper and lower rims of the orbital margin (Figure 1), Orbital breadth: horizontal distance between the dacryon and the orbital tubercle (Figure 2),

Orbital index: [orbital length/orbital breadth] *100

Percentage contribution of each bone: Length contributed by each bone $* 100$

Total length of the given orbital margin.

Inclusion criteria: orbits with normal morphology.

Exclusion criteria: orbits with fractured or broken margins.

Materials used for the study is that Dry skull specimens, Vernier caliper, Scale, Thread.

While the length and breadth of the orbit are determined by the use of a digital Vernier caliper placed between the corresponding anatomical landmarks as stated previously, the contributions of the frontal, maxilla and zygomatic bone towards making up the orbital margins cannot be determined likewise owing to their nonlinear morphology. In order to circumvent this shortcoming, a thread is run along the marginal contributions of the bones in question and the distance thus computed is measured against a scale with accuracy. 
Muthusamy AkshayaNarayanan, HannahSugirthabai Rajila Rajendran, et al.,A METRIC SCRUTINY OF THE ORBITAL MARGINS.

The results thus obtained are tabulated and crossanalysed with similar vernacular studies and the confluences and variations thus observed are elucidated. Analysis with the aid of software permits the interpretation of significant bilateral and dimorphic variations as well (Table 1).

Fig. 1: Showing the measuring procedure for orbital bredth.

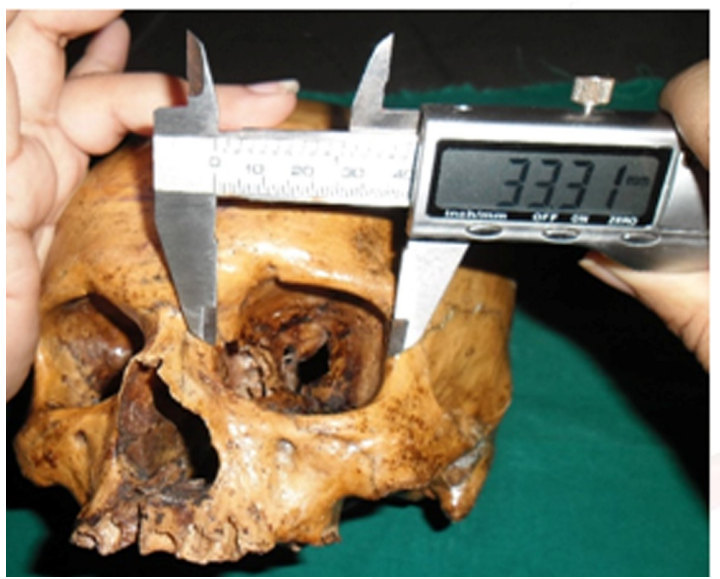

Fig. 2: Showing the measuring procedure for orbital length.

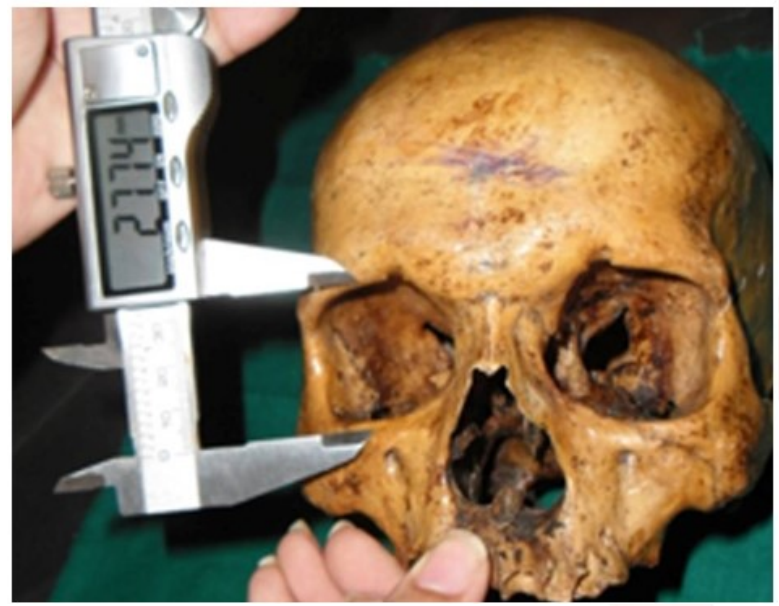

Fig. 3: Showing the measuring procedure for supraorbital notch.

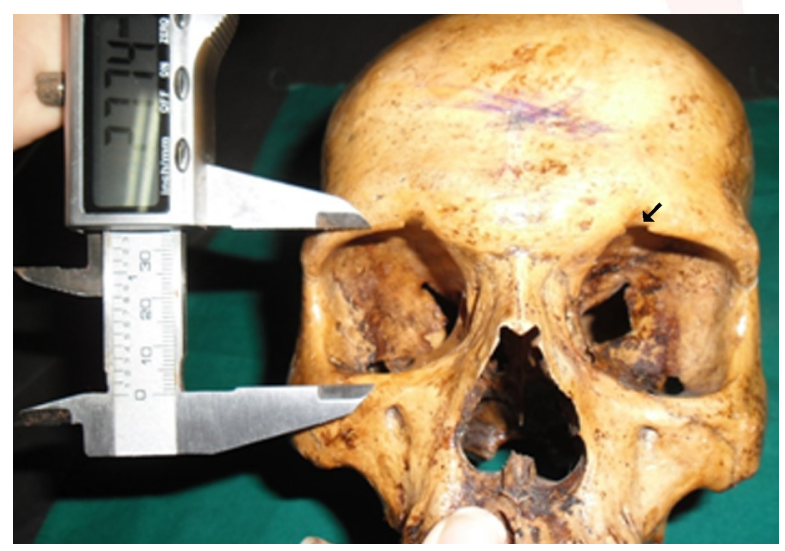

RESULTS

The average values of the territorial specimens are tabulated in table 1 and a predominance of
Microseme (45) category is found to be prevalent seconding which is the Mesoseme (26) category.

As far as recording the dimorphic variations prevalent in the anatomical makeup of the orbit goes, maxilla is found to be the major contributor as far as the medial orbital rim goes and no significant dimorphic variations are recorded.

While in the right orbit maxilla unanimously trials behind zygoma as far as its contribution towards making up the inferior margin go, the left orbit of the females defies this pattern of observation. In addition, the left zygomatic bone length of males showed significant variation.

An exceedingly overwhelming contribution towards making up the lateral orbital rim is found to be sourced out from the zygomatic bone. Thus it is justified to ascertain that the frontozygomatic suture, a cardinal topographical landmark, is located superiorly along the lateral orbital rim.

The superior margin is exclusively contributed by the frontal bone and no significant variations bilaterally are noticed. It is found that the left supraorbital notch/foramen in males displayed a significant inconsistency in its location along the supraorbital margin.

In both the right and left orbits, the breadth is found to exceed the length thus revealing that the orbit is horizontally wider and possesses a transverse axis which tends to out measure its vertical axis. Barring the left orbital index in females which conforms to the Mesoseme category, the others are subjected to be placed under Microseme.

A significantly greater contribution by the maxilla in making up the right medial margin over the left has been noted while the contrary is observed while assessing the frontal bone contributions. This corresponds to the bilateral variations as far as the medial margin is concerned. The preponderance of the zygoma as far as its contribution to the osseous anatomy of the orbital rim goes is duly noted to be larger in the right orbit.

A humongous contribution of the zygoma towards making up the lateral margin overshadowing the frontal bone's contribution is found to be nearly confluent bilaterally. 
Table 1: Orbital parameters (Average values).

\begin{tabular}{|l|c|}
\hline Orbital length & $31.26 \mathrm{~mm}$ \\
\hline Orbital breadth & $38.37 \mathrm{~mm}$ \\
\hline Orbital index & 81.47 \\
\hline $\begin{array}{l}\text { Frontal bone contribution towards } \\
\text { superior margin }\end{array}$ & $32.7 \mathrm{~mm}$ \\
\hline $\begin{array}{l}\text { Average distance of supraorbital } \\
\text { notch/foramen from medial extent of } \\
\text { superior margin }\end{array}$ & $5.92 \mathrm{~mm}$ \\
\hline $\begin{array}{l}\text { Frontal bone contribution towards } \\
\text { medial margin }\end{array}$ & $12.07 \mathrm{~mm}$ \\
\hline $\begin{array}{l}\text { Maxilla contribution towards the } \\
\text { medial margin }\end{array}$ & $13.81 \mathrm{~mm}$ \\
\hline $\begin{array}{l}\text { Maxilla contribution towards inferior } \\
\text { margin }\end{array}$ & $16.13 \mathrm{~mm}$ \\
\hline $\begin{array}{l}\text { Zygomatic bone contribution towards } \\
\text { the inferior margin }\end{array}$ & $17.29 \mathrm{~mm}$ \\
\hline $\begin{array}{l}\text { Zygomatic bone contribution towards } \\
\text { the lateral margin }\end{array}$ & $19.71 \mathrm{~mm}$ \\
\hline $\begin{array}{l}\text { Frontal bone contribution towards } \\
\text { the lateral margin }\end{array}$ & $4.90 \mathrm{~mm}$ \\
\hline Microseme & $45(60 \%)$ \\
\hline Mesoseme & $26(34.67 \%)$ \\
\hline \begin{tabular}{l} 
Megaseme \\
\hline
\end{tabular} & $4(5.33 \%)$ \\
\hline able $2:$ Cross tabulation - supraorbita
\end{tabular}

Table 2: Cross tabulation - supraorbital notch / foramen ( $R$ - Features * L - Features Cross tabulation).

\begin{tabular}{|c|c|c|c|c|}
\hline \multicolumn{2}{|c|}{$R$ - Features } & \multicolumn{2}{|c|}{ L- Features } & \multirow{2}{*}{ Total } \\
\cline { 3 - 5 } & $\begin{array}{c}\text { Supraorbital } \\
\text { notch }\end{array}$ & $\begin{array}{c}\text { Supraorbital } \\
\text { foramen }\end{array}$ & \\
\hline \multirow{2}{*}{$\begin{array}{c}\text { Supraorbital } \\
\text { notch }\end{array}$} & Count & 39 & 10 & 49 \\
\cline { 2 - 5 } & Row \% & 79.59 & 20.41 & 100 \\
\hline \multirow{2}{*}{$\begin{array}{c}\text { Supraorbital } \\
\text { foramen }\end{array}$} & Count & 11 & 15 & 26 \\
\cline { 2 - 5 } & Row \% & 42.31 & 57.69 & 100 \\
\hline \multirow{2}{*}{ Total } & Count & 50 & 25 & 75 \\
\cline { 2 - 5 } & Row \% & 66.67 & 33.33 & 100 \\
\hline
\end{tabular}

Fisher's Exact Test: Chi-Square Value: 10.626; Sig. : 0.002

Table 3: Orbital index -cross tabulation ( $R$ - Classification * L - Classification Cross tabulation).

\begin{tabular}{|c|c|c|c|c|c|}
\hline \multirow{2}{*}{ R-Classification } & \multicolumn{3}{|c|}{ L-Classification } & \multirow{2}{*}{ Total } \\
\cline { 2 - 5 } & Microseme & Mesoseme & Megaseme & \\
\hline \multirow{2}{*}{ Microseme } & Count & 36 & 6 & 1 & 43 \\
\cline { 2 - 5 } & Row \% & 83.72 & 13.95 & 2.33 & 100 \\
\hline \multirow{2}{*}{ Mesoseme } & Count & 11 & 8 & 5 & 24 \\
\cline { 2 - 5 } & Row \% & 45.83 & 33.33 & 20.83 & 100 \\
\hline \multirow{2}{*}{ Megaseme } & Count & 1 & 4 & 3 & 8 \\
\cline { 2 - 6 } & Row \% & 12.5 & 50 & 37.5 & 100 \\
\hline \multirow{2}{*}{ Total } & Count & 48 & 18 & 9 & 75 \\
\cline { 2 - 5 } & Row \% & 64 & 24 & 12 & 100 \\
\hline
\end{tabular}

Pearson's Chi-Square Test: Value: 21.348; Sig.: 0.000

While an increased dimension of the frontal bone which is the sole contributor towards the superior orbital rim is noted on the right, the right orbit also is found to display maximum variance as regards the same. The above stated postulation holds true as far as tracing the location of the supraorbital notch/foramen goes.

Nearly homogenous values are recorded bilaterally as far as gross orbital dimensions go indicating that the orbits are wider than they are longer uniformly.

An avant garde method of evaluation to crossreference the findings in the right and left has returned some interesting results (table 2). Of the 49 supraorbital notches noted in the right orbit only 39 were correspondingly found to be present in the left while the remaining ten were supraorbital foramen (Figure 3). Similarly, for the 26 supraorbital foramen noted in the right, only 15 were correspondingly same. Studies to test the symmetry aside, the presence of supraorbital notch is found to override the incidence of supraorbital foramina.

For the 43 microseme orbits recorded in the right only 36 were correspondingly microseme in the left while the rest sparing one were Mesoseme. Drawing similar comparisons, 8 out of 24 Mesoseme orbits in the right displayed an unflinchingly similar incidence in left while 11 and 5 orbits in the left corresponding to the aforementioned 24 fell under the category of Microseme and Megaseme respectively. A mere 3 for the corresponding 8 in the right orbit were found to be Megaseme while 1 and 4 other left orbits were microseme and Mesoseme respectively. A conflicting symmetry of orbital indices bilaterally is duly noted. A uniform finding though bilaterally is the escalated prevalence of microseme as is characteristic of the native population.

\section{DISCUSSION}

This study has facilitated an assiduous analysis of the orbital dimensions and hence the establishment of bilateral and dimorphic variations dictated by the same. The orbital index computed in this study is 81.47 which conforms to the category of Microseme. A study conducted in Himachal Pradesh by Maharana, SS et a [2] promulgated values conforming to the Microseme category. A pioneering study in Bathinda by Jaswinder Singh et al [3] deduced the mean orbital index to be 81.65 thus sectionizing the 
denizens of these north Indian province as belonging to Microseme. A confluent study in Ahmednagar, Maharashtra by Gosani, S.Net al [4] warranted the act of their aboriginal population (81.88) gaining ingression into the category of Microseme. A diligent probe to determine the orbital morphometry in Uttar Pradesh was conducted by Md.Tabrej Alam,et al [5] and a mean orbital index of 82.8 was deduced, adding to the occupants of Microseme category. A concurrent study conducted in the adult Bini skulls of Nigeria by Anibor, Ese et al[6] (78.54), and an analogous study in the Urhobo population of Nigeria by Ebeye.O.Aet al [7] (78.33) and a scrutiny of Sinhalese skulls (81.29) by Navneet et al [8] resonated the ubiquity of microseme as well. A scrutiny of dry skull specimens from Kenya by Munguti Jeremiah et al [9] with regards the orbital osteology (83.09), Mekala et al's [10] study of Bangalorean skulls(85.02) and examination of Turkish skulls(84.8) by V. Kaplanoglu et al[11] defied the sempiternal finding by establishing the overriding of microseme by Mesoseme.

As far as the gross dimensions of the orbits go, no significant distinction was noted between the two genders as well as between the two sides. Of importance is the fact that females showed a significantly $(p=0.008)$ higher value of orbital index as far as the left orbit goes when compared to their male counterparts. The right and left orbital indices for males and females were $81.54 \pm 5.39 \mathrm{~mm}$ and $82.65 \pm 6.36 \mathrm{~mm}$ (right) and $80.07 \pm 5.96 \mathrm{~mm}$ and $84.04 \pm 5.69 \mathrm{~mm}$ (left) respectively.

While Microseme was found to be the predominant type characterizing the aboriginal orbits, a novel method of perusal was carried out in the study to determine the bilateral homogeneity of the orbital indices for the same skull. Out of the 43 microseme orbits in the right, only 36 were correspondingly same in the left. Out of the remaining 7 left orbits, 6 were of Mesoseme category and 1 orbit was of Megaseme category. Prodding on similar lines, only 8 for the corresponding 24 in the right were Mesoseme in the left while 11 and 5 were Microseme and Megaseme respectively. On a confluent note, for 8 Megaseme right orbits, only 3 were correspondingly Megaseme in the left with 1 and 4 being Mesoseme and Microseme respectively.
Thus the homogeneity was maximum for the Microseme category (83.72\%) while Mesoseme (33.3\%) and Megaseme (37.50\%) were found to display bilateral dichotomy (Table 3).

In a riveting revelation, Sarah Fecht [12] has deduced that the orbital aperture shows an increase in size as the latitude progressively becomes higher. Her hypothesis was found to be veracious as upon comparative analysis of the values from Uttar Pradesh and Maharashtra, and here in Chennai, there was a progressive decline in orbital index; Bangalorean values being the only defiants of the same within the Indian subcontinent.

An avant-garde attempt was made in this study to characterize the anatomy of the bony orbital rims in humans though it had been previously carried out for animals only. The length of the frontal bone, the sole contributor towards the superior margin, displayed no significant dimorphic variations while bilateral variations were significant with right orbit recording greater values than the left. For 49 supraorbital notches in the right orbit, 39 were correspondingly homogenous (homogeneity $=79.59 \%$ ) in the left and for 26 supraorbital foramina in the right, 15 confluent occurrences were observed in the left (homogeneity $=57.69 \%$ ). While the incidence of supraorbital notch overrode that of foramen, both bilateral and dimorphic variations were recorded as far as its average distance from the medial extent of the superior margin goes, with right orbit having greater values and males having higher values as is concerned the left orbit, thus postulating the greater degree of its lateral displacement in the abovementioned categories. The supraorbital notch or foramina serves as a sapient guide for administration of supraorbital nerve block to accomplish regional anesthetic effect.

As far as the medial margin goes, maxilla was the greater contributor with the frontal bone closing on its heels and this displayed insignificant dimorphic variations. Bilaterally, though it was found that maxillary length and its percentage contribution was greater in the right orbit as opposed to the significantly higher frontal bone percentage in the left orbit. It has been reported that a predominant presence of maxilla in the medial margin results in posterior 
displacement of Weber's suture which provides a passage of transit for the infraorbital artery enabling it to ply to the nasal mucosa. When such a condition exists it is postulated that performing an osteotomy to reach the lacrimal sac is difficult during dacryocystorhinostomy due to the sheer thickness of maxilla. The anterior ethmoidal canal which is located at an average distance of $21 \mathrm{~mm}$ from the medial edge and the posterior ethmoidal canal which is located $14 \mathrm{~mm}$ posterior to it have orbital openings which help in demarcating the orbital areas into bulbar (part present anterior to anterior ethmoidal canal), retrobulbar (part present between anterior and posterior ethmoidal canals) and apex (part present posterior to posterior ethmoidal canal).

The lateral margin on the other hand was characterized by the overwhelming presence of zygomatic bone thus belittling the nearly insignificant contributions of the frontal bone and this finding displayed bilateral and dimorphic homogeneity. The frontozygomatic suture serves as a phare for surgeons and the reasons justifying the same are mentioned below.

The lateral orbital rim is recessed on its deep aspect approximately $0.75 \mathrm{~cm}$ above the rim margin to accommodate the lacrimal gland and this occasionally leads to a segmental fracture in this region. The insertion of a screw pin into the external angular process requires considerable care with its orientation if penetration into the orbit, or more significantly into the anterior fossa, is to be prevented. The pilot hole should be commenced $1.5 \mathrm{~cm}$ above the frontozy-gomatic suture and $0.5 \mathrm{~cm}$ behind the rim. The Whitnall's tubercle is present $11 \mathrm{~mm}$ inferior to frontozygomatic suture line and it is imperial to spare this during maxillary resection failing which damage to the Lockwood's ligament might cause inferior dystopia of the eyeball ultimately leading to diplopia. The pterion is $3.5 \mathrm{~cm}$ posterior to the frontozygomatic suture. The narrowest and weakest part of the lateral rim corresponds to the frontozygomatic suture and this is in continuity with the line of least resistance in the lateral wall which is situated at the junction of the greater wing of the sphenoid and the zygomatic bone. Consequently, separation at the frontozygomatic suture to a varying degree is a frequent finding following trauma in this region. Some authors imply that orbital exploration should be confined to a distance of $25 \mathrm{~mm}$ posterior to the frontozygomatic suture without damaging the structures coursing through the superior orbital fissure which is present $35 \mathrm{~mm}$ posterior to it [13]. The lateral orbital rim is in addition a component of the zygomaticomaxillary buttress and a profound comprehension of its anatomy is sapient in facial reconstruction surgeries to recreate the natural facial symmetry and morphology.

The inferior margin on the other hand presented the only significant bilateral variation with the males having greater values of zygomatic length as regards the left orbit(sig $=0.008)$. The zygomatic bone in addition was significantly greater grossly in the right orbit (sig $=0.021$ ). At the junction of the outer two-thirds and the inner one-third of the inferior orbital rim, there is a small depression which marks the origin of the inferior oblique muscle, the only extra ocular muscle which does not arise from the back of the orbit. This part of the inferior orbital rim is often fractured producing associated disruption of the muscle and subsequent diplopia.

The dynamic process of facial aging was studied by the American Society for Aesthetic Plastic Surgery and they professed that the orbital aperture width and area in both male and female subjects showed a significant increase with increasing age. In addition, findings suggested that the superior orbital rim receded with age in the medial region for both the genders alike. The inferior orbital rim receded significantly laterally in the female population, while the male subjects exhibited a recession of the entire inferior orbital rim.

\section{CONCLUSION}

The essential anatomy of the orbit has been analyzed and encapsulated to provide a repertoire that will be of use in the field of forensic anthropometry, to trace ethnic and evolutionary trends. It is of undisputable value to surgeons serving as beacons for the inception and pre/ post-operative management of surgical interventions. The establishment of a native orbital index is of cardinal value to spectacle manufacturers as it will allow them to design rim sizes 
suited for targeted niches depending on the preponderant orbital index type. The native population has revealed an overwhelming presence of Microseme category(45 out of 70) with Mesoseme being the second(26 out of 70).The maxillary preponderance as far as the medial margin goes reveals that the dacryocystorhinostomy procedures will be more endearing and will require greater dexterity on the surgeon's part. The left orbit of females has revealed that maxilla makes a greater contribution towards the inferior margin and that the average orbital index on this side is found to conform to the category of Mesoseme. The presence of supraorbital notch exceeded that of foramen. The hypothesis concerning the escalation of orbital index as one moves away from the equator has also been verified by crossanalysing the inferred value of orbital index (81.09) with other provincial values.

\section{Conflicts of Interests: None}

\section{REFERENCES}

[1]. FetouhAFathy, Mandour Dalia. Morphometric analysis of orbits in adult Egyptian skulls and its surgical relevance. Eur. J. Anat. 2014; 18 (4): 303-315

[2]. MaharanaSAgarwal R. Variations in the dimensions of the orbit in the adult human skull. International Journal of Recent Biochemistry. 2015; 3(3):20-22.

[3]. Kaur J, Yadav S, Singh Z. Orbit dimensions-A direct measurement study using dry skulls. J Acad Indus. 2012; 1(6).

[4]. Gosavi S, Jadhav S, Zambre B. A Study of Orbital Morphometry in Indian Dry Skulls. Asian Journal of Biomedical and Pharmaceutical Sciences. 2014; 4(29):23-25.
[5]. Alam D, Rai R, Singh M. Orbital Dimensions and orbital index of adult human dry skulls: A direct measurement study. International Journal of Scientific Research. 2016; 5(1).

[6]. AniborEighodae W. Orbital Index of Adult Binis in Edo State, Nigeria. International Journal of Forensic Medical Investigation. 2016; 2(1):17.

[7]. Ebeye O.A, Otikpo O., Orbital Index in Urhobos of Nigeria. IOSR Journal of Dental and Medical Sciences. 2013; 8(2):51-53.

[8]. Lal Navneet, Jon Cornwall, and George J. Dias. "Orbital Indices in a Modern Sinhalese Sri Lankan Population". Anatomy 10.3 (2016): 205-210. Web.

[9]. Jeremiah M, Pamela M, Fawzia B. Sex differences in the cranial and orbital indices for a black Kenyan population. International Journal of Medicine and Medical Sciences. 2013 Feb 28;5(2):81-4

[10]. D Mekala, R Shubha, Devi M R. Orbital Dimensions and Orbital Index: A Measurement Study on South Indian Dry Skulls. International Journal of Anatomy and Research. 2015; 3(3):1387-1391.

[11]. Kaplanoglu V, Kaplanoglu H, Toprak U, Parlak Ý, Tatar $Y$, Deveer $\mathrm{M}$ et al. Anthropometric measurements of the orbit and gender prediction with three-dimensional computed tomography images. Folia Morphology. 2014; 73(2):149-152.

[12]. Sarah Fecht, Bright-Sized: Skull Study Shows EyeSockets Have Grown Larger at Higher Latitudes ,www,scientificamerian.com

[13]. Patnaik VV, Sanju B, Rajan KS. Anatomy of the bony orbit-some applied aspects. J Anat Soc India. 2001 Jun; 50:59-67.

How to cite this article:

Muthusamy AkshayaNarayanan, HannahSugirthabai Rajila

Rajendran, Gnanasundaram Vaithianathan, Govindaraju

Soundararajan, Sundaramurthi Indumathi. A METRIC SCRUTINY

OF THE ORBITAL MARGINS. Int J Anat Res 2018;6(2.1):5065-5071.

DOI: 10.16965/ijar.2018.108 